\title{
Accessibility as Prerequisite for the Production of Individualized Aids Through Inclusive Maker Spaces
}

\author{
Hanna Linke ${ }^{(\star 凶)}$, Ingo K. Bosse, and Bastian Pelka \\ Faculty of Rehabilitation Research, University of Dortmund, Dortmund, Germany \\ Hanna. linke@tu-dortmund. de
}

\begin{abstract}
The article demonstrates by the example of persons with complex needs how to open up 3D-printing and peer production to everybody. It describes the requirements for accessibility and a competence-based scalable approach that empowers persons with complex needs to produce prototypes of products - such as assistive tools. In addition to discussing the systematics of the fields of action, the focus of the presentation will be the developed accessibility checklist and the tested products. Furthermore, first findings and products, such as a methodology for co-constructing products in a design thinking process and up taking results from social innovation, are described. General principles of accessibility for the design of maker spaces are presented, to sensitize the maker scene for this issue. These general principles are complemented by applicable standards, guidelines to be followed and supporting funding.
\end{abstract}

Keywords: Persons with disabilities $\cdot$ Accessible making $\cdot$ Peer production Social innovation $\cdot 3 \mathrm{D}$-printing

\section{Introduction}

Within the project SELFMADE, funded by the German Federal Ministry of Education and Research this project, the potentials of maker spaces and "making" for people with disabilities are explored. The project is linked to research and practice of assistive technology with a focus on participation in everyday life/leisure time, work and communication. It is not so much about assistive technology in the narrow sense than about the self-determined production of assistive aids.

For this purpose a maker space - a community space that offers tools and knowledge as well as exchange and learning for simply "making" things [1] - was set up in a Service Center for Augmentative and Alternative Communication (AAC) in Dortmund, Germany, where 12 persons with complex needs, including complex communication needs work daily. This project uses 3D-priniting as technology as well as the processes and platforms of social innovation. Other technologies often found in maker spaces such as melding, drilling or milling - are not used in this specific maker space in order to focus on the added value 3D-printing is offering for people with special needs. Starting with the group of persons with physical impairments and complex communication 
needs, SELFMADE tries to enable all persons with disabilities to design and produce products with a 3D-printer as well as sharing the designs with other people.

Persons with disabilities experience various barriers that prevent them from selfdetermined participation in social processes. Additional to structural barriers (e.g., accessibility), these include cognitive (e.g. easy language), emotional (e.g. repellent design of places), financial and technical barriers. In order to address these barriers in the research process the theoretical framework "capability approach" [2, 4] is used. This approach focusses the choices, which are necessary to initiate a process of social innovation through network building. Due to the used technology, the planning and producing of 3D-objects are also focused.

\section{The Aims of the Project}

The aims of the research and development project are:

1. The empowerment of persons with disabilities regarding the definition and production of individualized assistive tools.

2. The production of assistive tools in the areas work, everyday life/leisure time and communication.

3. The development, testing and research of a transferable problem-solving strategy, which is based on the principles of social innovation.

4. The preparation of a checklist for accessible maker spaces: How should maker spaces be designed to meet the minimum accessibility requirements.

Regarding accessibility, the project SELFMADE focuses on the following research questions:

1. How should maker spaces be spatially designed to implement minimum accessibility standards?

This article focuses on a checklist for accessible maker spaces and related tasks concerning the following main topics:

1. the text contents and text representation of information offers

2. architectural barriers, the accessibility of the building and the interior design as well as the availability of sanitary facilities or rest rooms

3. barriers in the product development, the using of CAD software and the production process by 3D-printing.

With this guideline and checklist, managers and planners from the maker scene should receive an instrument for planning and offering maker spaces with as few barriers as possible. In the maker space of the SELFMADE project, we were mainly concerned with $3 \mathrm{D}$ printing and describe this production process in this checklist, but it can also be applied to other ways of making.

First of all, this checklist gives an overview of the general notion of accessibility and the benefits of accessibility in maker spaces. General principles of accessibility initially 
serve as an overview and are substantiated by means of smaller checklists that can be used to check the accessibility.

This step includes the identification of barriers and addressing them, so these barriers will be dismantled. The checklists shows where barriers in a maker space can occur and how a maker space can become more accessible. The identification and addressing of barriers during the design and printing process enables as heterogeneous group as possible to be not excluded from the making process.

While providing the checklist the following questions need to be addressed in the project:

1. How should the connection to public transport be?

2. Which communication channels should be offered?

3. How is it possible to control $3 \mathrm{D}$ printers?

4. How modular are the products?

5. How can the "production process" be designed understandable?

6. How can the possible risks be well recognizable?

\section{Theoretical Framework}

The checklist for accessible maker spaces is an instrument to plan and offer maker spaces without excluding people with disabilities.

The underlying definition of accessibility refers to the German disability equality law:

BGG§4: “...if they (things) are accessible and usable for disabled people in the usual way, without any particular difficulty and in principle without outside help".

The following principles describe the extent of accessibility:

- Foot-Wheel-Principle: Are the building and the offers accessible and usable for wheelchair users as well as pedestrians?

- Two-sense principle (channel rule/multi-sensory principle): Is information perceivable by at least two of the senses (seeing and additionally hearing or touching)?

- KISS Rule: Information is offered using the "Keep It Short and Simple" method.

Additionally, principles of "Universal Design" are considered to enable as many people as possible to take part in a maker space:

1. Principle - Wide usability: The design should be usable and marketable for people with different abilities.

2. Principle - Flexibility in use: The design should support a wide range of individual preferences and possibilities.

3. Principle - Simple and intuitive use: Use of the design should be easy to understand and independent of the user's experience, knowledge, language skills or current concentration.

4. Principle - Sensory information: The design should provide the user with the necessary information effectively, regardless of the user's environment or sensory capabilities. 
5. Principle - Fault tolerance - The design minimizes risks and the negative consequences of accidental or unintentional actions.

6. Principle - Low physical effort: The design should be efficient and comfortable to use with a minimum of fatigue.

7. Principle - Size and space for access and use: Provide adequate space and space for access, accessibility, manipulation and use regardless of the user's size, posture or flexibility.

\section{Methodology}

Regarding the methodology the product development is based on a repetitive researchand development cycle, often used in the context of social innovation, in order to address accessibility in the project SELFMADE.

The products are developed in an iterative cycle, based on the principles of Design Thinking [3].

The Design Thinking is composed of six steps (Fig. 1).

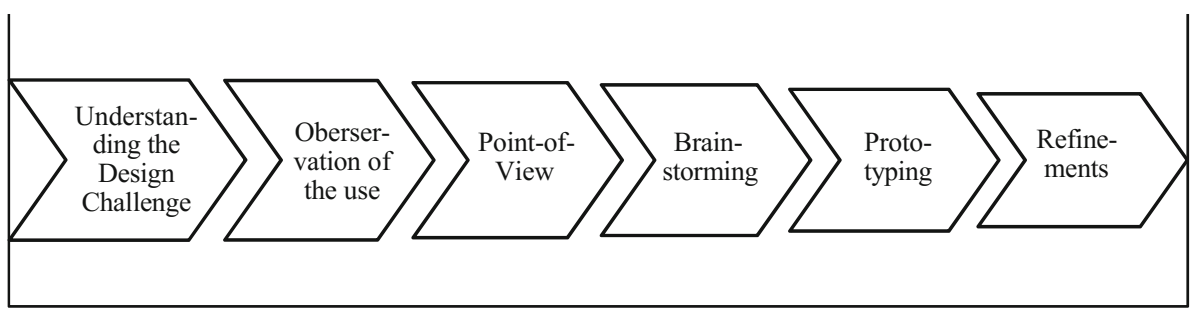

Fig. 1. Design thinking process cf. Schallmo [3], p. 18

In this cycle three product lines are successively tested by persons with disabilities. During this process experiences, gained while developing each "product", are considered in the design of the derived product. Thus, in an early stage of the development a "finished" product is available, the complexity increases across the product generations and a learning process of the participants (e.g. technical procedures, individual cultures, potentials and restrictions) takes place. As a result, the process of identification of needs (which can be addressed with photonic procedures), the implementation competence of the target group as well as the quality of the products, increases with each product generation.

Each of these "products" is identified on the basis of a needs analysis. In the needs analysis the project uses a User Centered Design approach, which is linked with a Design Thinking process and can be seen as both: as a process of creating social innovations and as a social innovation itself. This allows to identify, define, create and test the designed products in a Co-Creation process by persons with disabilities for persons with disabilities. 


\section{Prerequisite Results}

Due to the fact, that the projects still runs until the end of August 2018 it is just possible to present the results at its current intermediate status.

The focus is placed on the accessibility checklist and the tested products.

Every chapter of the accessibility checklist for maker spaces deals with the following topics:

1. The implementation of the general principles: How is it possible to implement general principles of accessibility with small changes?

2. General legal requirements: Further information on the general legal requirements and on support agencies in their area to implement accessibility.

3. Financial support: In the case of extensive conversion measures or changes

4. Quick check: A short checklist at the end of each chapter can be used to quickly check your maker space for accessibility.

While testing for accessibility it is necessary to examine barriers which might occur during the visit in a maker space and even earlier, from access to the building to barriers which can appear during the making process. This already begins "on the way to the maker space" as soon as one tries to find out something about a maker space on a homepage, in a flyer or on other channels, which are used to present the maker space in public.

In order to make texts comprehensible to as many people as possible, they should be based on specific principles in terms of content, structure and graphic representation. That can be principles of easy-to-read, additional information in capital letters and Braille or touchable logos, which can be printed in 3D.

The next step is the accessibility to public transport or the parking lot situation at the maker space.

Possible barriers include missing elevators at train stations, missing signage on the way to the maker space, or lack of parking lots.

During the project, it turned out to be one of the most crucial barriers that allows only a few people with disabilities to regularly visit a maker space. Reasons for this are the lack of access to public transport, the complex organization of transport services or lack of funds.

Arriving at the maker space, additional areas emerge that need to be checked for accessibility. This includes the accessibility of the building, the respective interior design, sanitary facilities and possibly additional premises.

The financing and design of accessible buildings is clearly defined and differentiated depending on whether it is a private or public building. Organizers of maker spaces often have little influence on that, but if they point out possible barriers, interested people can adjust to it.

Ultimately, the process of making should be checked for accessibility.

That includes the developing of the product, the printing process and the adjusting of hardware like the 3D-printers and the CAD software.

In order to address accessibility in the printing and development process, the following steps of design thinking can be used. This includes the appropriate documentation: 
1. Understanding: The first step focuses on the understanding of the problem, this leads to an appropriate question, which defines the needs and challenges of the project. Data sources: photo documentation and written collection, composed from workshops and Open Spaces.

2. Observation: In order to gain important insights and to define the status quo an intensive search and field observation follows. Data sources: field notes and written collection, composed from workshops/Open Space.

3. Point-of-view: the individual needs are clearly defined in one question: Data source: documentation of the brainstorming question.

4. Brainstorming to develop and visualize different concepts. Data source: Different visualized concepts with the program Design Spark Mechanical.

5. Prototyping: In order to test and to demonstrate the ideas, first simple prototypes are developed and tested by the target group. Data source: Prototypes.

6. Refinement: On the basis of the insights gained from the prototype, the concept is further improved and refined until an optimal, user-centered product is developed. This iteration step can be applied on all previous steps.

\subsection{Five Levels of Competence}

According to the different competences of the target group we distinguish between five competence levels in order to enable everyone to use a 3D-printer and to print their own product.

Communication via Assistants or Assistive Technology, Almost No Movement Abilities, No ICT Skills. A shelf is displaying products that could be printed with a 3D-printer. The selection was made in workshops with persons with similar impairments. Persons with disabilities express which object they like to receive and assistants initiate the printing process.

Communication Only via Assistants or Assistive Technology, Basic Movement Abilities, No Ict Skills. A SIM card is attached to each object and can be inserted in a $3 \mathrm{D}$-printer. We designed the $3 \mathrm{D}$ printer in a way that enables most users to initiate the printing process.

Basic ICT Skills. Users with basic ICT skills can click on pre-selected models for printing.

Advanced ICT Skills. For advanced users we offer CAD software that enables the alteration of existing models or design from sketch.

Advanced ICT Skills, Basic Communication Skills. Users become tutors in 3D printing in peer education processes.

To increase the competence level it is necessary to consider if the user needs different keyboards or other peripheral device, changeable font size or contrasts or the possibility of reading function. In addition during the SELFMADE project we developed several 
adjustments for the 3D-printer like a risk minimizing door or buttons for a better operation.

This approach is particularly attractive for the globally networked maker spaces because it has the potential to reach new target groups by making it as accessible as possible.

\section{References}

1. Dougherty, D.: The maker movement. Innov.: Technol. Gov. Glob. 7(3), 11-14 (2012)

2. Nussbaum, M.: Frontiers of Justice: Disability, Nationality, Species Membership. Harvard University Press, Cambridge (2006)

3. Schallmo, D.R.A.: Design Thinking erfolgreich anwenden. Springer Gabler, Wiesbaden (2017). https://doi.org/10.1007/978-3-658-12523-3

4. Sen, A.: Ökonomie für den Menschen: Wege zu Gerechtigkeit und Solidarität in der Marktwirtschaft, München, Wien (2000)

5. Story, M.: Maximizing usability: the principles of universal design. Assist. Technol. 10, 4-12 (2010)

Open Access This chapter is licensed under the terms of the Creative Commons Attribution 4.0 International License (http://creativecommons.org/licenses/by/4.0/), which permits use, sharing, adaptation, distribution and reproduction in any medium or format, as long as you give appropriate credit to the original author(s) and the source, provide a link to the Creative Commons license and indicate if changes were made.

The images or other third party material in this chapter are included in the chapter's Creative Commons license, unless indicated otherwise in a credit line to the material. If material is not included in the chapter's Creative Commons license and your intended use is not permitted by statutory regulation or exceeds the permitted use, you will need to obtain permission directly from the copyright holder. 